\title{
Isolation of Methicillin-resistant Staphylococcus aureus (MRSA) using a flocked swab elution transport system; with identification by PCR
}

\author{
Aus Molan \\ Department of Research and in-vitro Diagnostics/Abacus ALS/New Zealand
}

\begin{abstract}
Admission screening is an essential part of the control of methicillin-resistant Staphylococcus aureus (MRSA). Using collection systems that maintain viability and have high organism recovery, combined with accurate and rapid laboratory testing is critical for the success of a screening program. This review evaluates the performance of PCR testing, particularly the Roche LightCycler MRSA test, in the detection of MRSA. In addition, the clinical performance of the Copan Elution swab (ESwab) is reviewed. Several commercial molecular tests developed for use in detecting MRSA colonisation have been evaluated against culture methods, and all appear to provide high sensitivity, good specificity, and the potential for rapid reporting of MRSA colonisation. The new LightCycler MRSA advanced test is another of these assays with rapid detection time and high sensitivity. Compared with the BD GeneOhm MRSA assay, this new test also appears to have improved test specificity. The ESwab has an equal or even better recovery of bacteria, including MRSA, compared with other swab systems, via culture, and PCR. The advantages of using the ESwab are: the use of a liquid Amies medium allowed multiple uses (both culture, and PCR can be performed with one collected sample); and, specimens from multiple sites can be pooled into the same liquid transport media which limits the test costs and broadens the screening capabilities. However, there are limited publications on the clinical performance of the ESwab and more research is required on this new swab transport system.
\end{abstract}

Keywords: Bacterial culture, infection control, mass screening, Methicillin-Resistant Staphylococcus aureus microbial viability, MRSA, Polymerase Chain Reaction, PCR.

\section{INTRODUCTION}

Staphylococcus aureus is an opportunistic pathogen carried as a commensal organism on the skin and nares of approximately $30 \%$ of the normal population [1]. The potential to cause a broad spectrum of diseases that are fatal within 30 days on infection has been reported in approximately $34 \%$ of cases [1]. S.aureus can rapidly adapt to the selective pressure from antibiotic treatments, which has resulted in the emergence and spread of methicillin-resistant S.aureus (MRSA) strains. The mecA gene, responsible for this resistance to methicillin and to other $\beta$-lactam antibiotics, is located on a large mobile genetic element called the Staphylococcal Cassette Chromosome mec (SCCmec). The mecA gene encodes for an altered penicillin binding protein, PBP2a or PBP2, which has a very low affinity for all $\beta$-lactam antibiotics allowing normal synthesis of the peptidoglycan layer to occur, even in the presence of these antibiotics [2]. Numerous MRSA stains have emerged and spread worldwide, and SCCmec has been acquired be different lineages of methicillin-senstive $S$. aureus [3]. To date, five SCCmec types (I-V) have been distinguished, and several variants of these SCCmec types have been described [3].

Healthcare associated infections caused by MRSA have become an important issue for healthcare facilities worldwide due to high rates of infection, mortality, and high costs of treatment [4]. In addition, community-associated MRSA has spread in the past few years [5], feeding the pipeline of infection in hospitals [6], and underscoring the need for comprehensive infection control programs. In 2008, recommendations from published guidelines of several governmental, professional, and public health organisations were summarised to help institutions prioritise and implement their MRSA transmission prevention efforts [7]. Factors that determine the success of a MRSA prevention program include: the patient population tested [8]; the efficiency of a collection systems to maintain viability and recovery of organisms [19]; the sensitivity and speed of laboratory testing [9]; and the speed of result determination [8-10].

Real-time PCR detection of MRSA has been shown to significantly reduce time to detection (under two hours of laboratory time) compared with standard culture tests $[8,10,11]$. This improves the efficiency of control and management strategies, leading to a significant reduction in MRSA transmission [8, 10, 11]. For MRSA detection, swabs are the most commonly used sampling device. These systems must maintain organism viability during transit while allowing maximal organism recovery during laboratory testing. Given the increasing frequency of transport delays due to cost containment measures, consolidations, and services being 
shifted to centralised or reference laboratories, robust transport systems are becoming increasingly relevant. Recently, Copan have developed a modified liquid Amies elution swab (ESwab) suitable for MRSA screening.

The purpose of this review is to evaluate the performance of PCR, particularly the Roche LightCycler (LC) MRSA test, in the detection of MRSA in comparison to current culture methods. In addition, the clinical performance of the Copan ESwab for the maintenance of viability of MRSA is also discussed.

\subsection{The need and emergence of PCR technology}

\section{PCR AND MRSA}

The most efficient approach to control of endemic MRSA remains controversial [12]. Several authorities have suggested that screening on admission to intensive care units and subsequent patient isolation may decrease the risk for MRSA cross-infection [13]. Rapid screening tests may further improve MRSA control, because traditional microbiological methods for MRSA screening are slow [14]. Delays in receiving screening results means either that negative patients remain isolated for too long or that positive patients remain a hidden reservoir for cross-infection. With the availability of rapid molecular MRSA screening methods, determining their value in daily practice is of great importance.

A cohort study undertaken in Geneva described a reduction in MRSA infections in a medical intensive care unit (ICU), when a rapid molecular screening test was combined with pre-emptive isolation of all admissions until a negative result was obtained [10]. The same trend was reported during an observational cohort study, set in a 19-bed mixed medical and surgical adult critical care unit in southwest England. This study found a significant reduction (65\%) in the rate of MRSA transmission in a critical care unit when admission screening by culture was replaced with a same-day polymerase chain reaction (PCR) test [11]. Possible explanations include earlier use of decolonisation regimens, earlier use of appropriate antibiotics, earlier isolation, and better compliance with hand hygiene procedures. Moreover, Cunningham et al. found the reduction in turnaround time gained using the PCR test is substantial, since presumptive results are obtained early in the ICU stay, when nursing and medical care is most intensive. It could be reduced even further if the PCR test is made available at weekends [11].

Most importantly, analysis of the recent literature indicates that only tests permitting capture of $>70 \%$ of potential MRSA patient isolation days will provide a positive impact for a MRSA control program within one year from its implementation in a setting of modest MRSA colonisation [31]. Only real-time PCR assays have the capability in sensitivity and result-reporting time to offer that result.

\subsection{Roche LightCycler MRSA test}

A five site study [27] compared the performance of the Roche LC MRSA advanced test to the BD GeneOhm MRSA test (an FDA-cleared diagnostic assay for rapid detection of MRSA) and culture for detection of MRSA found in surveillance nasal swabs.

Compared with the BD GeneOhm MRSA assay, the Roche LC has improved test specificity with direct culture (91.7\% and 96.4\%, respectively) and enrichment culture (91.8\% and $96.8 \%$, respectively). Positive percent agreement (relative sensitivity) with directly plated and enrichment culture for the LC MRSA advanced test and the BD GeneOhm MRSA assay did not differ significantly. There was little variation in the positive percent agreement between individual sites (91.4 to 100\%).

High sensitivity of any MRSA surveillance test is desirable since the goal of a MRSA program is to rapidly detect all those colonized with this potential pathogen, even if nasal detection results from identifying DNA no longer associated with viable organisms in the nares. However, improved specificity can be particularly important when performing a large number of assays in a population with a modest level of asymptomatic MRSA colonisation to minimise false positive test results that can lead to inappropriate patient isolation.

Also, the Roche LC MRSA test required less labour time than the comparator assay and per-formed as well as culture: approximately $1.5 \mathrm{~min}$ of technologist time per sample for the Roche PCR assay compared to 3 min for the BD GeneOhm MRSA assay and 1 to 2 min for direct culture.

More recently, a multi-site study conducted in New Zealand [32] evaluated the clinical performance of the Roche LightCycler PCR assay in the detection of MRSA in comparison to routine chromogenic agar culture method (BD BBL CHROMagar II). From this study, the MRSA incidence among volunteers was $2.6 \%$ (culture) and $4.8 \%$ (PCR). The $2.2 \%$ higher yield obtained by the PCR method was statistically significant $(P=0.04)$. This increase in sensitivity incurs an additional cost (per test, the PCR assay was 2.5 times more expensive). However, the PCR method had a much faster result turnaround time (2-3 hours) compared to culture (48 hours) while both methods had comparable sample hands on time $(1.65 \mathrm{~min}$ and $1.20 \mathrm{~min}$, respectively. The authors conclude that it wise to introduce PCR for MRSA screening. 
transport system; with identification by PCR

\section{COPAN ESWAB}

\subsection{Clinical development}

Because of their convenience, swab systems with transport media are often used to collect and transport specimens of various types. Swab tips should be prepared with material that collects sufficient specimen material, is nontoxic to microorganisms, maintains viability in conjunction with the transport medium, and releases specimen material efficiently onto agar media [15]. Cotton, Dacron, and rayon swabs absorb bacteria in their surface, thereby, enmeshing bacteria in their dense fibre matrix and compromising their release [16].

A new type of swab system has recently been introduced in a growing number of laboratories [17]. The Copan ESwab consists of a sterile package containing two components: a pre-labelled polypropylene screw-cap tube with conical shaped bottom filled with $1 \mathrm{ml}$ of Liquid Amies transport medium and a specimen collection swab which has a tip flocked with soft nylon fibre [18]. This swab is prepared by spray-on flocked fibre technology has been developed for transport of bacteria and viruses [19]. This technology provides stronger capillary action and strong hydraulic uptake of liquids, which should result in better specimen collection [20]. This design also provides more efficient release of specimen material and, therefore, less entrapment of specimen than occurs with typical rayon or Dacron fibre-tipped swabs [20]. Organisms present in the specimen remain close to the surface and when placed in transport medium (modified liquid Amies), elute completely and immediately [22]. The increased efficiency of this system has been demonstrated in vitro [23] and in vivo on volunteers [19]. Another advantage of the ESwab system is the use of a liquid Amies medium and its multiple delayed uses: both culture, rapid antigen testing and PCR can be performed with one collected sample. According to the product insert, the ESwab may be stored at room temperature $\left(20-25^{\circ} \mathrm{C}\right)$ or in the refrigerator $\left(4-8^{\circ} \mathrm{C}\right)$ and processing may be delayed up to maximally 48 hours [18].

\subsection{Characteristics of the ESwab}

With electron microscopy, Human and Jones [23] showed that bacteria adhere to the fibres of the flocked swab while this was more difficult to demonstrate in conventional swabs; presumably because bacteria were absorbed onto the surface and enmeshed/trapped within the dense fibre matrix. The brush-like flocked nylon fibre traps bacteria by capillary action (Table 1).

Human and Jones [23] also showed by means of radioactive tracers, that flocked swabs release much more particles ( $92 \%$ of the initial inoculums) than conventional cotton wool/rayon swabs (30\% of the initial inoculum). A couple of studies confirmed this better release of bacteria because: the zero-time counts of ESwab were higher than of the other swab systems tested [15, 24]; and, cycle threshold values of RT-PCRs were similar to that of the initial inoculum when using ESwab [24].

Since the ESwab system provides increased organism release, this would theoretically mean that a greater number of all organisms including pathogens and commensal microorganisms would be recovered. Infections with a low bacterial load might be detected more efficiently if collected with such a swab with enhanced releasing capability [22].

Another advantage of the flocked swab in liquid medium is that every inoculated plate receives the same inoculum. Conventional swabs, when used to plate a number of agar plates, result in less inoculum on the last plate [23].

A previous study of Moore et al. [25] showed that dry flocked swabs could keep viral RNA stable up to 15 days storage at room temperature. Moreover, the amount of nucleic acid released from the swab is highly consistent over the time [25]. This study indicates that flocked swabs can reliably be used for molecular testing.

\subsection{MRSA}

According to the study of Smismans et al. [26], the recovery of MRSA with the Copan ESwab exceeded that of the Venturi Copan swab system by a factor of at least 3.6. Moreover, the ESwab had a 1 to $2 \log$ higher recovery of microorganisms on MRSA chromogenic agar plate than the Copan Stuart liquid transystem [27]. This means that the theoretic chance of detecting MRSA increases too.

The MRSA ESwab collection kit of nares, throat and axilla was fully concordant with the traditional Amies agar gel swab of nares in terms of positive MRSA samples with culture and pre-enrichment culture on CHROMagar MRSA and PCR [28]. This pooling of specimens from multiple sites limits the test costs and broadens the screening capabilities [27]. Martens et al. [29] validated the pooled MRSA ESwab from nose, throat and perineum for use instead of the nose, throat and perineum samples taken by a double Copan swab [cepheid collection device]. ESwab proved to be a valid alternative as input for the Xpert MRSA ${ }^{\circledR}$ assay run on the GeneXpert ${ }^{\circledR}$ system (Cepheid) [29].

Since culture and PCR can be performed on the same sample, thanks to the $1 \mathrm{ml}$ liquid Amies medium of the ESwab, sampling bias is reduced and the need for taking several clinical samples is eliminated [27, 29].

Molan et al. [32], aimed to determine the MRSA isolation rates from swabs transported in semi-solid media (Copan Venturi Transystem) and liquid-based media (Copan ESwab). They tested 810 swabs and found that 
both swab types performed almost equally in maintaining the viability of MRSA during the study. While the elution swab was approximately double the price of the Venturi counterpart, the liquid phase allows the pooling of samples and multiple testing to be performed from one swab specimen.

\subsection{Acceptance criteria for Swabs}

The performance of swab systems can be evaluated by means of the CLSI document M40-A: Quality Control of Microbiological Transport Systems; Approved Standard [30]. When using the qualitative roll-plate method (Fig. 1), a viability study with a swab system is considered acceptable when there are $\geq 5$ CFU following the storage time from the plate with the same dilution as that with a zero-time count of $+/-300 \mathrm{CFU}$ [30]. When using the quantitative swab elution method (Fig. 2), a viability study with a swab system is considered acceptable if there is no more than a $3 \log 10(+/-10 \%)$ decline in CFU between the zero-time CFU count and the CFU of the swabs that were stored [30].

In the Copan ESwab product insert [18], all CLSI acceptance criteria (quantitative swab elution method and qualitative roll-plate method) were met for Haemophilus influenzae, Streptococcus (S.) pyogenes, S. pneumoniae, Pseudomonas aeruginosa, Neisseria gonorrhoeae (24 hrs storage), Peptostreptococcus anaerobius, Bacteroides fragilis, Fusobacterium (F.) nucleatum, F. necrophorum, Propionibacterium acnes, Prevotella melaninogenica, Enterococcus faecalis, Staphylococcus aureus, S. agalactiae, Clostridium (C.) sporogenes, C. perfringens, Peptococcus magnus after storage for $48 \mathrm{hrs}$ at $4-8^{\circ} \mathrm{C}$ and $20-25^{\circ} \mathrm{C}$ [15].

Smismans et al. [26] reported a 4-and 9-times higher recovery with ESwab compared with the Venturi swab in the roll-plate and the swab elution method respectively. This means that the inoculum on the Venturi swab has to be 4- to 9-fold higher to reach a similar detectable growth as with the ESwab [26].

In the study of Giambra and Castriciano [27], the sensitivity limit was better with the Copan ESwab than with Stuart liquid transystem. This means that MRSA could be detected up to higher dilutions with ESwab compared with Stuart liquid transystem for both culture and molecular techniques [27].

As is generally known, temperature has a significant effect on preservation of microorganisms in transport devices [30]. Previous studies have shown that cold temperatures are superior to room temperature. Room temperature is not the optimal holding temperature for maximum preservation of microbiological samples [30].

There is no CLSI standard for the evaluation of overgrowth in swab transport systems at room temperature [30]. At present, none of the commercial available transport devices are able to adequately inhibit microbial overgrowth at room temperature [30].

\subsection{Cost impact}

The ESwab system is more expensive than traditional Venturi swabs, \$69.69 and \$30.62 respectively (estimated calculations per pack of 50 in \$NZD exc. GST). When the number of swabs to be taken can be reduced (by for e.g. performing MRSA PCR and culture with the same swab, or be pooling samples), it could be possible that the resulting cost equals the present one. Moreover, the better performance of the ESwab would increase the MRSA detection rate. This would include a shorter length of stay and a reduced hospital cost for nosocomial MRSA infections. Therefore, the introduction of the expensive ESwab might be economical overall.

III. FIGURES AND TABLES

\begin{tabular}{|c|c|c|}
\hline & Flocked swab & Cotton/rayon swab \\
\hline Particle release & $92 \%$ of initial inoculum & $30 \%$ of initial inoculum \\
\hline Method of bacterial uptake & Adhering by capillary action & Absorbed and enmeshed \\
\hline Consistent inoculum per culture plate & Constant & Decrease \\
\hline Electron microscopy images & & \\
\hline & &
\end{tabular}

Table 1. Comparison of the physical and microscopic features of the flocked and cotton/rayon swabs in terms of particle release; method of bacterial uptake; and continuous inoculum release per culture plate Summarised from Human and Jones [23]. 


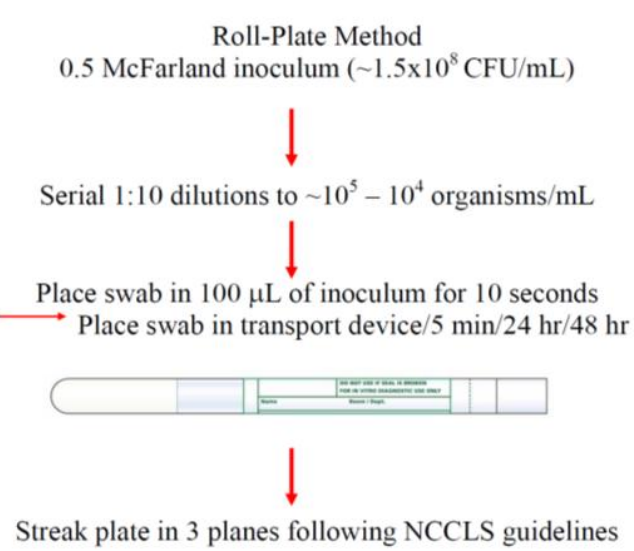

Figure 1. Schematic Diagram of Roll Plate Method The performance of swab systems can be evaluated by means of the CLSI document M40-A: Quality Control of Microbiological Transport Systems; Approved Standard [30] - qualitative roll-plate method.

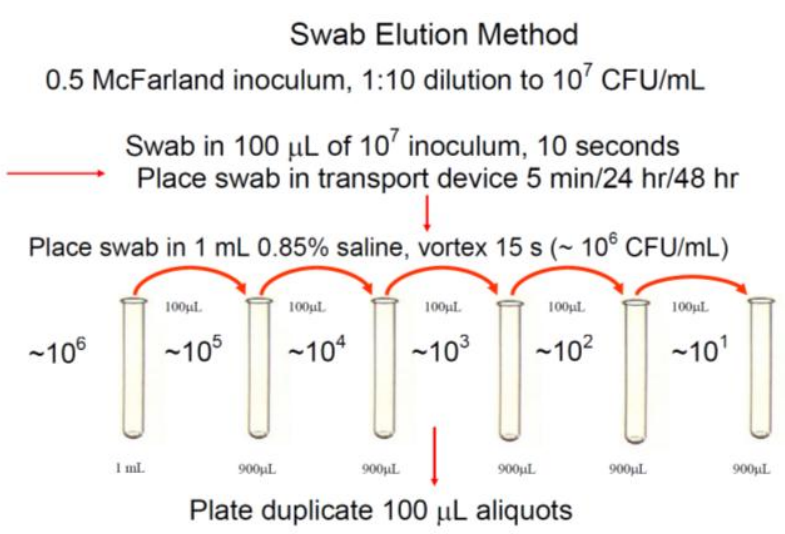

Figure 2. Schematic Diagram of Swab Elution Method. The performance of swab systems can be evaluated by means of the CLSI document M40-A: Quality Control of Microbiological Transport Systems; Approved Standard [30] - quantitative swab elution method.

\section{CONCLUSIONS}

From the available literature, it is evident that implementation of PCR methods in the detection of MRSA lead to a reduction in the subsequent transmission of MRSA. The new Roche LC MRSA advanced test performed with sensitivity equal to that of the BD GeneOhm MRSA assay, and both outperformed the sensitivity and time to reporting of directly plated culture. The LightCycler MRSA advanced test appeared to have improved specificity compared to the BD GeneOhm MRSA assay. Also, the Roche LC test had hands-on technologist processing time comparable to that of directly plated culture and thus can provide very rapid results with no increase in laboratory personnel.

The Copan ESwab has an equal or even better recovery of MRSA compared with other swab systems, via culture, and PCR. Two advantages of the ESwab are evident. Firstly, the use of a liquid Amies medium and its multiple uses (both culture and PCR can be performed with one collected sample). This reduces sampling bias is reduced and the need for taking several clinical samples is eliminated. Secondly, specimens from multiple sites can be pooled into the liquid transport media which limits the test costs and broadens the screening capabilities.

Until present, there are no guidelines that have implemented this new type of swab transport system. The swab system was introduced in 2008, literature data are still limited (albeit many posters) and more research is warranted.

\section{REFERENCES}

[1] M Kuehnert, D Kruszon-Moran, H Hill, G McQuillan, S McAllister, G Fosheim, et al. Prevalence of Staphylococcus aureus Nasal Colonization in the United States, 2001-2002. Journal of Infectious Diseases, 193(2), 2006, 172-179.

[2] M Phino, S Filipe, H De Lencastre, and H Tomasz, A. Complementation of the Essential Peptidoglycan Transpeptidase Function of Penicillin-Binding Protein 2 (PBP2) by the Drug Resistance Protein PBP2A in Staphylococcus aureus. Journal of Bacteriology, 183 (22), 2006, 6525-6531.

[3] R Deurenberg, C Vink, S Kalenic, A Friedrich, C Bruggeman, and E Stobberingh. The molecular evolution of methicillin-resistant Staphylococcus aureus. Clinical Microbiology and Infection, 13(3), 2007, 222-35. 
[4] J Engemann, Y Carmeli, S Cosgrove, V Fowler, M Bronstein, S Trivette, et al. Adverse Clinical and Economic Outcomes Attributable to Meticillin Resistance among Patients with Staphylococcus aureus Surgical Site Infection. Clinical Infectious Diseases, 36(5), 2003 592-598. S Cosgrove, G Sakoulas, E Perencevich, M Schwaber, A Karchmer, and Y Carmeli. Comparison of mortality associated with methicillinresistant and methicillin-susceptible Staphylococcus aureus bacteremia: a meta-analysis. Clinical and Infectious Diseases, 36(1), 2003, 5359.

[6] R Klevens, M Morrison, J Nadle, S Petit, K Gershman, S Ray, et al. Invasive Methicillin-Resistant Staphylococcus aureus Infections in the United States. The Journal of the American Medical Association, 298(15), 2007, 1763-1771.

[7] D Calfee, C Salgado, D Classen, K Arias, K Podgorny, D Anderson, et al. Strategies to Prevent Transmission of Methicillin-Resistant Staphylococcus aureus in Acute Care Hospitals. Infection Control and Hospital Epidemiology 1, 2008, S62-80.

[8] L Peterson, O Liesenfeld, C Woods, S Allen, D Pombo, P Patel, et al. Multicenter Evaluation of the LightCycler Methicillin-Resistant Staphylococcus aureus (MRSA) Advanced Test as a Rapid Method for Detection of MRSA in Nasal Surveillance Swabs. Journal of Clinical Microbiology, 48(5), 2010, 1661-1666.

[9] S Paule, M Mehta, D Hacek, T Gonzalzles, A Robicsek, and L Peterson. Chromogenic media vs real-time PCR for nasal surveillance of methicillin-resistant Staphylococcus aureus: impact on detection of MRSA-positive persons. American Journal of Clinical Pathology, 131(4), 2009, 532-539.

[10] S Harbarth, C Masuet-Aumatell, J Schrenzel, P Francois, C Akakpo, G Renzi, et al. Evaluation of rapid screening and pre-emptive contact isolation for detecting and controlling methicillin-resistant Staphylococcus aureus in critical care: an interventional cohort study. Critical Care, 10(1), 2006, R25.

[11] R Cunningham, P Jenks, J Northwood, M Wallis, S Ferguson, and S Hunt. Effect on MRSA transmission of rapid PCR testing of patients admitted to critical care. Journal of Hospital Infection, 65, 2006, 24-28.

[12] J Cepeda, T Whitehouse, B Cooper, J Hails, K Jones, F Kwaku, L Taylor, S Hayman, B Cookson, S Shaw, et al. Isolation of patients in single rooms or cohorts to reduce spread of MRSA in intensive-care units: prospective two-centre study. Lancet, 365, 2005, 295-304.

[13] B Rubinovitch, and D Pittet. Screening for methicillin-resistant Staphylococcus aureus in the endemic hospital: what have we learned? Journal of Hospital Infection, 47, 2001, 9-18.

[14] D Diekema, K Dodgson, B Sigurdardottir, and M Pfaller. Rapid detection of antimicrobial-resistant organism carriage: an unmet clinical need. Journal of Clinical Microbiology, 42, 2004, 2879-2883.

[15] K Van Horn, and I Rankin. Evaluation and comparison of two Stuart's liquid swab transport systems tested by the CLSI M40 method. European Journal of Clinical Microbiology and Infectious Diseases, 26, 2007, 583-586.

[16] M Österblad, H Järvinen, K Lönnqvist, S Huikko, P Laippala, J Viljanto, H Arvilommi, and P Huovinen. Evaluation of a New Cellulose Sponge-Tipped Swab for Microbiological Sampling: a Laboratory and Clinical Investigation. Journal of Clinical Microbiology, 41, 2003, $1894-1900$.

[17] V Saegeman, J Flamaing, J Muller, W Peetermans, J Stuyck, and J Verhaegen. Clinical evaluation of the Copan ESwab for methicillinresistant Staphylococcus aureus detection and culture of wounds. European Journal of Clinical Microbiology and Infectious Diseases, 8, 2011, 943-9.

[18] Copan. Copan Liquid Amies Elution Swab (ESwab) Collection and Transport System. (Product Insert \& How to Use Guide). Copan Innovation, Brescia, Italy, 2010. Retrieved July 28, 2011, from: http://www.copanswabs.com/products/eswab

[19] P Verhoeven, F Grattard, A Carricajo, B Pozzeto, and P Berthelot. . Better detection of S. aureus nasal carriage by use of flocked nylon swabs. Journal of Clinical Microbiology, 48, 2010, 4242-4244.

[20] Copan. Why flocked swabs are superior to fiber wrapped swabs and foam swabs and how they can improve infectious disease diagnosis. Copan Innovation, Brescia, Italy, 2010. Retrieved from: http:// www.mls.be/.../Why-Flocked-Swabs-are-Superior-to-Fiber-and-Foam.pdf

[21] K Van Horn, C Audette, K Tucker, and D Sebeck. Comparison of 3 swab transport systems for direct release and recovery of aerobic and anaerobic bacteria. Diagnostic Microbiology and Infectious Disease, 62, 2008, 471-473.

[22] D Halstead, J Abid, C Laing, H Davis, L Moore, K Carter, C Lewis, A Veydt, P McCartt, and P McKitrick, P. Evaluation of the Copan flocked ESawb vs Copan dual swabs for anteriot nares specimen collection for MRSA/ORSA using the Xpert MRSAATM assay. Poster presented at the 26th Clinical Virology Symposium, April 28 2010, Daytona Beach, Florida, USA. Retrieved from: http://pascv2010.ivdnews.net/public/show_abstract/821.html

[23] R Human, and G Jones. A new concept for transporting clinical material on flocked swabs in liquid Amies medium. C-107. Paper presented at the 106th American Society for Microbiology General Meeting, May 222006 . Retrieved from: http://www.copanswabs.com/studies/download.php?id=48

[24] J Davidson, M DeFields, A Bergstrome, C Pienaar, L Martinez, and M Kelly. Evaluation of Flocked Nylon Swabs (Copan ESwab) for Detection of Bacterial Pathogens by Culture and PCR. Poster presented at the American Society for Microbiology Toronto, Canada, May 21 2007. Retrieved from: http://www.copanswabs.com/studies/main.php?topic=3\&year=2007

[25] C Moore, S Corden, J Sinha, and R Jones. Dry cotton or flocked respiratory swabs as a simple collection technique for the molecular detection of respiratory viruses using real-time NASBA. Journal of Virology Methods, 153, 2008, 84-89.

[26] A Smismans, J Verhaegenb, A Schuermansc, and J Fransa. Evaluation of the Copan ESwab transport system for the detection of methicillinresistant Staphylococcus aureus: a laboratory and clinical study. Diagnostic Microbiology and Infectious Disease, 65, 2009, 108-111.

[27] A Giambra, and S Castriciano. Evaluation of the ESwab for MRSA screening with real-time PCR, BD GeneOhm and culture. Poster presented at the 5th European Meeting on Molecular Diagnostics. Scheveningen, The Hague, Netherlands, 2007. Retrieved from: http://www.copanswabs.com/studies/download.php?id=26

[28] S Silbert, L Carballo Menezes, J Monteiro, M Celle Ribeiro, S Pereira and A Pignatari. Screening of MRSA: a comparison between one and multiple sites sample collection using the new Eswab MRSA collectiion kit. Poster American Society for Microbiology, 109th General Meeting, Philadelphia, PA, May 18 2009. Retrieved from: http://www.copanswabs.com/studies/download.php?id=719

[29] K Martens, H De Beenhouwer, J Frans, A Van den Abeele, R Cartuyvels, and G Coppens. Evaluation of eSwabTM for surveillance of MRSA by Xpert MRSATM and culture on pooled samples. Poster presented at the 19th ECCMID, Helsinki, May 16 2009. Retrieved from: http://www.copanswabs.com/studies/download.php?id=706

[30] Clinical and Laboratory Standards Institute (CLSI). Quality control of microbiological transport systems; approved standard, M40-A, Vol. 23, 2007, No. 34.

[31] L Peterson, and D Diekema. To screen or not to screen for methicillin-resistant Staphylococcus aureus. Journal of Clinical Microbiology, 48, 2010, 683-689. [Point-Counterpoint].

[32] A Molan, M Nulsen, G Thomas. Methicillin-resistant Staphylococcus aureus (MRSA): isolation from nasal and throat swabs transported in liquid or semisolid media; identification by PCR compared with culture. New Zealand Journal of Medical Laboratory Science, 67, 2013, 816. 